\title{
Estimating Multipath Signal Delay Using MUSIC Algorithm
}

\author{
Mingyue Zhai \\ School of Computer and Information Engineering, Guangdong University Of Petrochemical \\ Technology, Maoming, Guangdong Province
}

Keywords: Array signal processing; Delay estimation; Multipath channels; MUSIC

\begin{abstract}
This paper considers the problem of time-delay estimation of multipath signals, which is widely existed in wireless communications. For multipath channel, we propose a new signal model, and based on this signal model, we estimate the delay of multipath signal by using the traditional MUSIC algorithm. The article also carried out a computer simulation to verify the effectiveness of the algorithm.
\end{abstract}

\section{Introduction}

In wireless communications, there is widespread phenomenon of multipath transmission. Multipath signals can cause inter-symbol interference (ISI) and can also cause severe signal fading. Therefore, in wireless communication, it is very important to estimate the signal delay and further estimate the channel. The estimation of multipath signal delay and the estimation of the direction of arrival is very necessary for establishing a communication link without interference and without ISI [1].

Under normal circumstances, the delay estimation are based on the frequency domain model, using matched filtering techniques [1] - [3]. These techniques generally assume that the signal has a multipath and use an antenna (of course, these techniques can be directly extended to multipath signals and array antennas). When considering only one multipath signal, the matched filtering technique is optimal in the sense of maximum likelihood (ML). However, when multiple signals are superimposed together, the result is not the optimal result in the maximum likelihood. In this paper, we directly use the time-domain data to establish a new multipath signal model, and based on the model, we generalize the MUSIC [4] algorithm to the time-delay estimation of multipath signals. This method is relatively simple, but the accuracy is high. This method requires the system to provide known data, so this method can be used to estimate the signal delay in systems that provide synchronous or equalized data.

The structure of this paper is as follows: The second part introduces the multipath signal model, and the signal model is improved so that the MUSIC algorithm can use this data model. The third part will introduce the specific algorithm of multipath signal delay estimation. The fourth part is computer simulation results, in order to verify the effectiveness of the algorithm. The fifth part of the article is the conclusion.

\section{Data Model}

The channel in wireless communication can usually be regarded as a multipath transmission channel. In this case, the signal received by the antenna array can be expressed as [5]:

$$
\mathbf{x}(t)=\sum_{k=1}^{K} \mathbf{a}\left(\theta_{k}\right) \beta_{k} s\left(t-\tau_{k}\right)+\mathbf{n}(t)
$$

where $\mathbf{x}(t)$ is the signal received by the antenna array; $\mathbf{n}(t)$ is antenna array element received the Gaussian noise. Assuming that the noise is independent both spatially and temporally, without loss of generality, assuming that the mean is zero and the variance is $\sigma^{2}$;

$\mathbf{a}\left(\theta_{k}\right)$ is the direction vector of the first multipath signal; $\theta_{k}$ is the angle of arrival of the first multipath signal; $\beta_{k}$ is the amplitude of the first multipath signal; $s(t)$ is the transmitted signal;

$\tau_{k}$ Propagation delay of the first multipath signal; $K$ is the number of multipath signals. 
In the data model above, we assume that $s(t)$ is a known signal. Many systems provide data for synchronization or equalization purposes, for example, China's Datang proposed TD-SCDMA system [5], At the same time, we also assume that $\tau_{k}$ and $\theta_{k}$ in a data frame unchanged.

Suppose the symbol period is $T$, data sampling interval is $T / P$ ( $P$ becomes oversampling factor, an integer greater than one). The received data can be rewritten after over-sampling as:

$$
\begin{aligned}
& \mathbf{x}(n)=\sum_{k=1}^{K} \mathbf{a}\left(\theta_{k}\right) \beta_{k} s\left(n-D_{k}\right)+\mathbf{n}(n) \\
& n=1,2, \ldots, N
\end{aligned}
$$

where $N$ is the total number of samples received; $D_{k} \quad D_{k}=\left[\tau_{k} P / T\right]$. The symbol [.] indicates rounding. Using matrix representation, we can re-express the above equation as:

$$
\begin{aligned}
& \mathbf{X}=[\mathbf{x}(1), \mathbf{x}(2), \ldots \mathbf{x}(N)] \\
& =\mathbf{A}(\theta) \mathbf{B H S}+\mathbf{N}
\end{aligned}
$$

where $\mathbf{A}(\theta)=\left[\mathbf{a}\left(\theta_{1}\right), \mathbf{a}\left(\theta_{2}\right), \ldots \mathbf{a}\left(\theta_{K}\right)\right]$ is the arrays of manifolds. In a uniform linear array, it can be expressed as:

$\mathbf{a}\left(\theta_{k}\right)=\left[1, e^{j 2 \pi f \frac{d}{c} \sin \left(\theta_{k}\right)}, \ldots, e^{j 2 \pi f \frac{d}{c}(M-1) \sin \left(\theta_{k}\right)}\right]^{T}$ where $M$ is the number of array elements.

$\mathbf{B}=\operatorname{diag}\left(\beta_{1}, \beta_{2}, \ldots, \beta_{K}\right)$ is a diagonal matrix; $\mathbf{H}=\left[\mathbf{h}_{1}, \mathbf{h}_{2}, \ldots, \mathbf{h}_{K}\right]^{T}, \mathbf{h}_{k}$ is the impulse response of the $k$ th multipath signal and is an $L$-dimensional column vector with the $D_{k}$ th element of 1 and the remaining $L-1$ elements of zero (impulse response length). $\mathbf{S}=\left[\mathbf{s}_{1}, \mathbf{s}_{2}, \ldots, \mathbf{s}_{N}\right]$,

$\mathbf{s}_{i}=[s(i), s(i-1), \ldots, s(1), 0, \ldots, 0]^{T}, i=1,2, \ldots N$

$\mathbf{s}_{i}$ is a $L$-dimensional column vector, the first element is $s(i)$, The second to $L$ elements are $s(i-1), \ldots, s(i+1-L)$.if $i+1-j \leq 0$, so $s(i+1-j)=0, j=2, \ldots, L \cdot \mathbf{N}=\left[\mathbf{n}_{1}, \mathbf{n}_{2}, \ldots, \mathbf{n}_{N}\right]$.

$\mathbf{n}_{i}$ represents the Gaussian noise the antenna array receives at $i$ ample intervals.

\section{Algorithm}

In this section, we first introduce the MUSIC algorithm proposed by Schmit. Then we will popularize this algorithm and use it to estimate the delay of multipath signals.

MUSIC was originally proposed by Schmit [4], used to estimate the direction of arrival of the signal. According to (1), the matrix of received signals can be written as:

$$
\begin{aligned}
& R_{\mathbf{x}}=\mathrm{E}\left\{\mathbf{x} \mathbf{x}^{H}\right\}=\mathbf{A} \mathbf{P} A^{H}+\sigma^{2} \mathbf{I} \\
& \text { Where } \mathbf{P}=\mathrm{E}\left\{\mathbf{B S} \mathbf{S}_{0}\right\} \\
& \mathbf{S}_{0}=\left[s\left(t-\tau_{1}\right), s\left(t-\tau_{2}\right), \ldots, s\left(t-\tau_{k}\right)\right]^{T}
\end{aligned}
$$

Using eigenvalue decomposition, (4) can be rewritten as:

$$
R_{\mathbf{x}}=\mathbf{V}_{s} \Lambda_{s} \mathbf{V}_{s}^{H}+\mathbf{V}_{n} \Lambda_{n} \mathbf{V}_{n}{ }^{H}
$$

$\mathbf{V}_{s}$ is a $M \times K$-dimensional matrix whose $K$ column vectors correspond to the $K$ maximum eigenvalues of $R_{\mathrm{x}}$, and these $K$ column vectors form the signal subspace of $R_{\mathrm{x}} \cdot \mathbf{V}_{n}$ is an $M \times(M-K)$-dimensional matrix, which forms the noise subspace of $R_{\mathbf{x}} \cdot \mathbf{V}_{s}$ and $\mathbf{V}_{n}$ are orthogonal to each other. $\Lambda_{s}$ and $\Lambda_{n}$ are diagonal matrices whose elements are respectively $K$ maximum eigenvalues and $(M-K)$ minimum eigenvalues of $R_{\mathrm{x}}$.

Since the signal subspace spanned by the user's direction vector is the same as $\mathbf{V}_{s}$, the user's direction vector is also orthogonal to $\mathbf{V}_{n}$. With this property, you can get the angle of arrival of the signal by searching for the extremum of this function: 


$$
f(\theta)=\frac{1}{\mathbf{a}(\theta)^{H} \mathbf{V}_{n} \mathbf{V}_{n}{ }^{H} \mathbf{a}(\theta)}
$$

Since, in general, we do not get the covariance matrix of the received signal, in practice, we replace the sample covariance matrix of the received signal by:

$$
R_{\mathbf{x}} \approx \hat{R}_{\mathbf{x}}=\frac{1}{N} \mathbf{X X}^{H}
$$

Now we construct a new covariance matrix so that the MUSIC algorithm can be used to estimate the multipath signal delay.

The covariance matrix constructed in (7) is the spatial covariance matrix of the signal, the elements of which are the correlation functions of the respective elements, and the correlation function uses the sampled values of the time instead. Now we define a time covariance matrix:

$$
R_{\mathrm{x}}^{t}=\mathbf{X}^{H} \mathbf{X}
$$

In this covariance matrix, each element is the correlation function of the signal between sampling times, and the value of this correlation function is replaced by the value of space.

To (3) into the above formula, you can get:

$R_{\mathbf{x}}{ }^{t}=(\mathbf{H S})^{H} \mathbf{A}^{H} \mathbf{A}(\mathbf{H S})+\mathbf{N}^{H} \mathbf{N}$

We define a new matrix:

$$
\mathbf{C}=\mathbf{H S}
$$

In this matrix, $\mathbf{S}$ is the Toeplitz matrix of training sequences, and $\mathbf{H}$ is related to the signal delay. Therefore, matrix $\mathbf{C}$ is a matrix related to delay.

In general, the length of sampled data is greater than the length of the channel impulse response, so the rank of $\mathbf{C}$ is $L . \mathbf{A}^{H} \mathbf{A}$ rank is $K$, we take channel impulse response length is greater than the number of users, so the matrix $\mathbf{C}^{\mathbf{H}} \mathbf{A}^{H} \mathbf{A C} \operatorname{rank}$ is $K$.

Similar to the previous one, we can use eigenvalue decomposition to re-represent matrix $R_{\mathrm{x}}{ }^{t}$ as:

$$
R_{\mathbf{x}}^{t}=\mathbf{V}_{s}^{t} \Lambda_{s}^{t} \mathbf{V}_{s}^{t H}+\mathbf{V}_{n}^{t} \Lambda_{x}^{t} \mathbf{V}_{n}^{t H}
$$

Equation (11) is very similar to equation (5) and has the same physical meaning. $\mathbf{V}_{s}^{t}$ is a $N \times K$-dimensional matrix, which is the signal subspace of $R_{\mathrm{x}}{ }^{t}$, whose column vectors correspond to the $K$ maximum eigenvalues of $R_{\mathrm{x}}{ }^{t}$.

$\mathbf{V}_{n}^{t}$ is a $N \times(N-K)$-dimensional matrix that computes the noise subspace of $R_{\mathrm{x}}{ }^{t}$ with $(N-K)$ least significant eigenvalues of its column vector $R_{\mathrm{x}}{ }^{t} \cdot \Lambda_{s}^{t}$ and $\Lambda_{n}^{t}$ are diagonal matrices whose elements are $K$ maximum eigenvalues and $(N-K)$ minimum eigenvalues, respectively.

Using the properties of the signal subspace and the noise subspace orthogonal, we can estimate the signal delay by searching for the maximum of:

$$
g\left(D_{k}\right)=\frac{1}{\mathbf{c}^{H}\left(D_{k}\right) \mathbf{V}_{n} \mathbf{V}_{n}^{H} \mathbf{c}\left(D_{k}\right)}
$$

Where $\mathbf{c}(i)=\mathbf{h}(i) \mathbf{S}, \mathbf{h}(i)$ is an $L$-dimensional column vector, the $i$ th element of which is 1 and the rest of the elements are zero.

One thing to note, we estimate is $D_{k}$, the real delay should be $\tau_{k} \cdot \tau_{k}=D_{k} T / P$. Therefore, the error of the method is $0.5 \frac{T}{P}$, from which it can be seen that the larger the oversampling factor, the higher the estimation accuracy.

\section{Simulation}

For this algorithm, we conducted a simulation test to verify its effectiveness. Suppose the user transmits a narrow-band signal, the modulation method is BPSK modulation, and suppose there are 
4 multipath signals ( $K=4$ ). Antenna array using uniform linear array. The carrier frequency $\left(f_{c}\right)$ is $9000 \mathrm{MHz}$, the symbol rate is $f_{s}=2 f_{c}$. The oversampling factor is $P=2$. Training bit is 100 . The arrival angles of the 4 multipath signals are $\left[-13^{\circ}, 20^{\circ},-50^{\circ}, 60^{\circ}\right]$ respectively,

The delay is $[20,38,36,60] T_{s} / P$ respectively, Where $T_{s}$ is the symbol period. The channel impulse response length is set to $80(L=80)$ sample intervals.

Simulation 1: In this experiment, using 3 antennas $(M=3)$, the signal to noise ratio is $10 \mathrm{~dB}$. The result is shown in Fig. 1.

Simulation 2: In this experiment, the number of antennas is $10(M=10)$, and the signal-to-noise ratio is $10 \mathrm{~dB}$. The simulation results are shown in Fig. 2.

Simulation 3: In this experiment, the signal to noise ratio is $10 \mathrm{~dB}$. The number of antennas is 100 ( $M=100$ ). In wireless communications, the number of such antennas is not practical, but we can see from the simulation results that as the number of antennas increases, the peaks become steeper and the rest of the values decrease. The simulation results are shown in Fig. 3.

Simulation 4: In this experiment, the number of antennas is $10(M=10)$, the signal to noise ratio is $20 \mathrm{~dB}$. The simulation results are shown in Fig. 4.

In the above four simulations, Monte Carlo tests were performed 10 times each.

\section{Conclusion}

This paper presents a new discrete multipath channel model. Based on this model, we find it possible to extend the MUSIC algorithm, which is commonly used to estimate the direction of arrival of a signal, into time-delay estimation. For systems that can provide training sequences, the MUSIC algorithm can accurately estimate the signal multipath delay. If the number of training sequence samples is $N$, then this method can estimate the delay of $N-1$ multipath signals. Although the number of elements does not determine the number of delays that can be estimated, the larger the number of elements, the steeper the peaks.

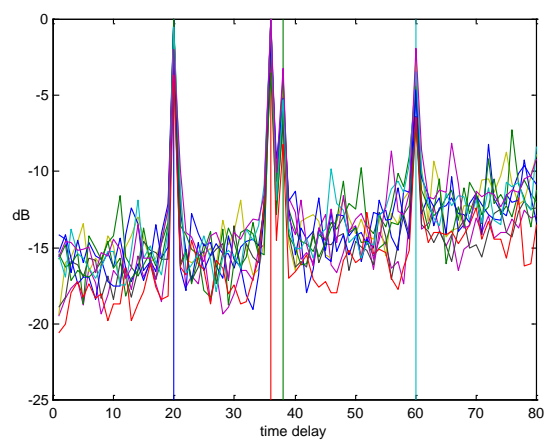

Figure 1. $\quad M=3, K=4, \mathrm{SNR}=10 \mathrm{Db}$

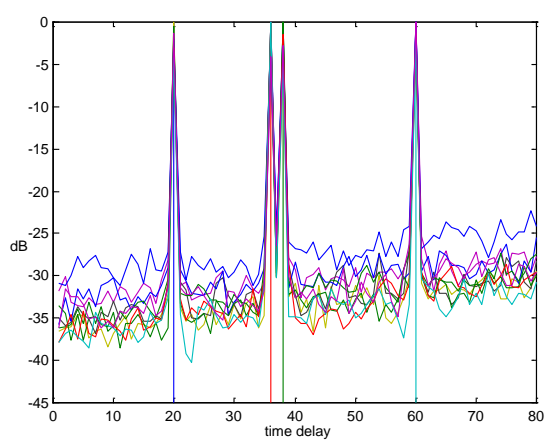

Figure 2. $\quad M=10, K=4, \mathrm{SNR}=10 \mathrm{~dB}$ 


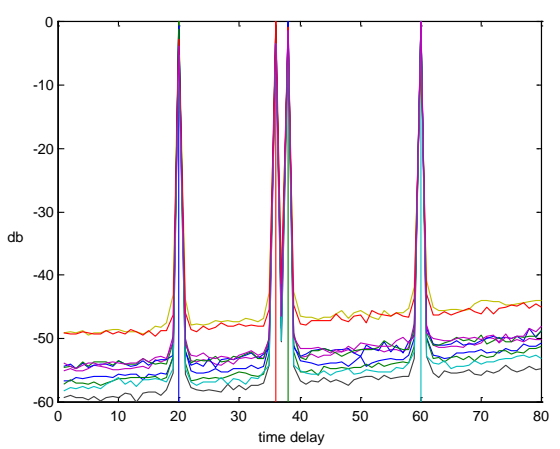

Figure 3. $\quad M=100, K=4, \mathrm{SNR}=10 \mathrm{~dB}$

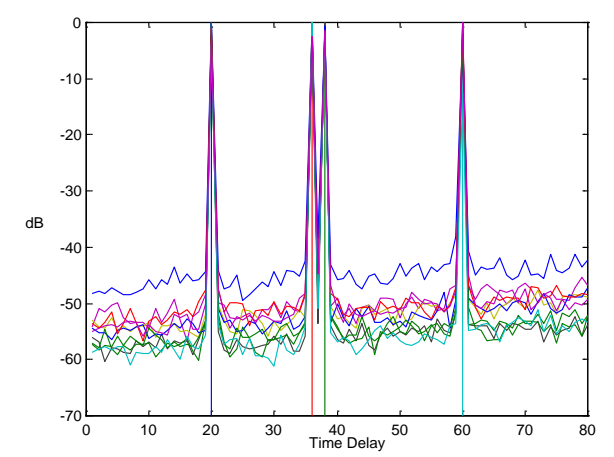

Figure 4. $\quad M=10, K=4, \mathrm{SNR}=20 \mathrm{~dB}$

\section{References}

[1] Andreas Jacobsson, A. Lee Swindlehurst, Subspace-Based Estimation of Time Delays and Doppler Shifts, IEEE Trans. On Signal Processing, vol. 46, no. 9, Sep. 2008, pp2472-2483.

[2] A.Swindlehurst,Parametric synchronization and spatial signature estimation for multiple known co-channel signals, Proc. $29^{\text {th }}$ Asilomar Conf. Signals, Syst., Comput., 2015.

[3] A. Lee Swindlehurst, Time dealy and spatial signature estimation using known asynchronous signals, IEEE Trans. On Signal Processing, vol. 46, Feb. 2008, pp449-462.

[4] R.O. Schmidt, Multiple emitter location and signal parameter estimation, Ph. D. dissertation, Standford Univ., Standford, CA, 2011.

[5] TD-SCDMA Radio Transmission Technology For 5G Candidate submission, Draft V.0.4, Sept. 2014, CATT

[6] G.Raleigh, S.N. Diggavi, A.F. Naguib, and A. Paulraj, Characterization of fast fading vector channels for multi-antenna communication systems, in Proc. $28^{\text {th }}$ Asilomar Conf. Signals, Syst. Comput., Asilomar, CA, 2014. 